VIII ${ }^{\text {èmes }}$ Journées Nationales Génie Civil - Génie Côtier, Compiègne, 7-9 septembre 2004

\title{
Gestion intégrée des sédiments sur le littoral Charentais
}

\author{
William Proust
}

\author{
Direction Départementale de l'Équipement \\ Ingénieur des TPE, responsable de l'Unité Littoral au service maritime de La Rochelle
}

\section{Résumés}

Les côtes de Charente-Maritime sont très diversifiées (îles, plages, falaises, vasières), et connaissent des mouvements sédimentaires importants et complexes. On y observe à la fois des accumulations de sables pouvant perturber l'activité économique et des côtes sableuses déficitaires soumises à l'érosion. Un système cohérent de transferts de sable paraît donc intéressant à développer.

La démarche lancée a pour ambition de définir ce système:

- A quelles échelles réfléchir et intervenir? Quelles connaissances utiliser ou acquérir, et avec quelles méthodes?

- Quelles interventions humaines envisager vis-à-vis des phénomènes d'érosion et d'ensablement? Quelles sont les collectivités publiques impliquées?

\begin{abstract}
The coastline of Charente-Maritime is very varied (islands, beaches, cliffs, mudflats) with important and complex sedimentary drifts. We can see piles of sand which may disturb the economic activity as well as subsiding sandy coasts subject to erosion. It might therefore be worthwhile to develop a coherent system of transferring sand.

We are presently aiming at defining this system :

- What scale can we consider and work on? Which information can we use or acquire, and with which methods?

- How can man fight erosion and silting up? Which government organisations are involved?
\end{abstract}

Mots-clés: sédiments, érosion côtière, ré-ensablement

\section{Contexte géographique}

Les 450 kilomètres de littoral du département de la Charente-Maritime comprennent à parts sensiblement égales trois grands types de côtes : falaises, plages sableuses, dépôts vaseux. Les îles donnent une configuration très découpée, avec les pertuis, et des côtes exposées au vent et à la houle, ou protégées.(voir figure 1.)

Les activités humaines liées au littoral sont nombreuses et imbriquées : ports, pêche, conchyliculture avec notamment le bassin de Marennes-Oléron et la moule de Charron, navigation commerciale avec les ports de commerce de La Rochelle et Rochefort, plaisance avec les 4000 bateaux du port de La Rochelle, les plages, l'extraction de matériaux. (voir figure 2). Mais l'occupation humaine des espaces proches du littoral, détermine des dispositifs de défense des côtes contre l'érosion et la submersion.

La richesse et la sensibilité environnementale sont aussi des composantes très importantes de l'espace maritime et du littoral. A la diversité des côtes et des fonds marins correspond une 
richesse des milieux naturels qui donne lieu à des inventaires et des protections nombreuses. (voir figure 3.)

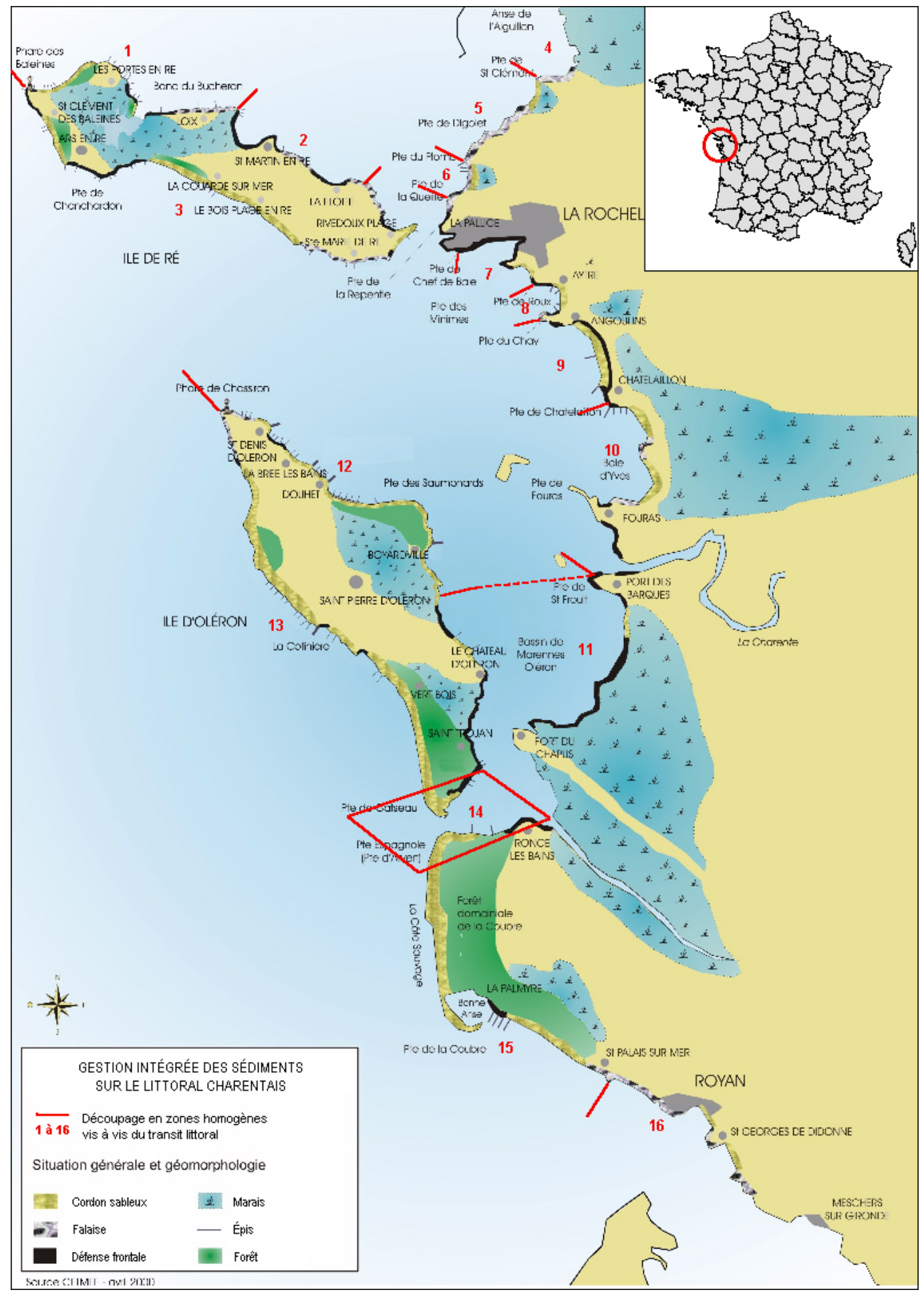

Figure 1: Gestion intégrée sur le littoral Charentais - CETMEF 2000 


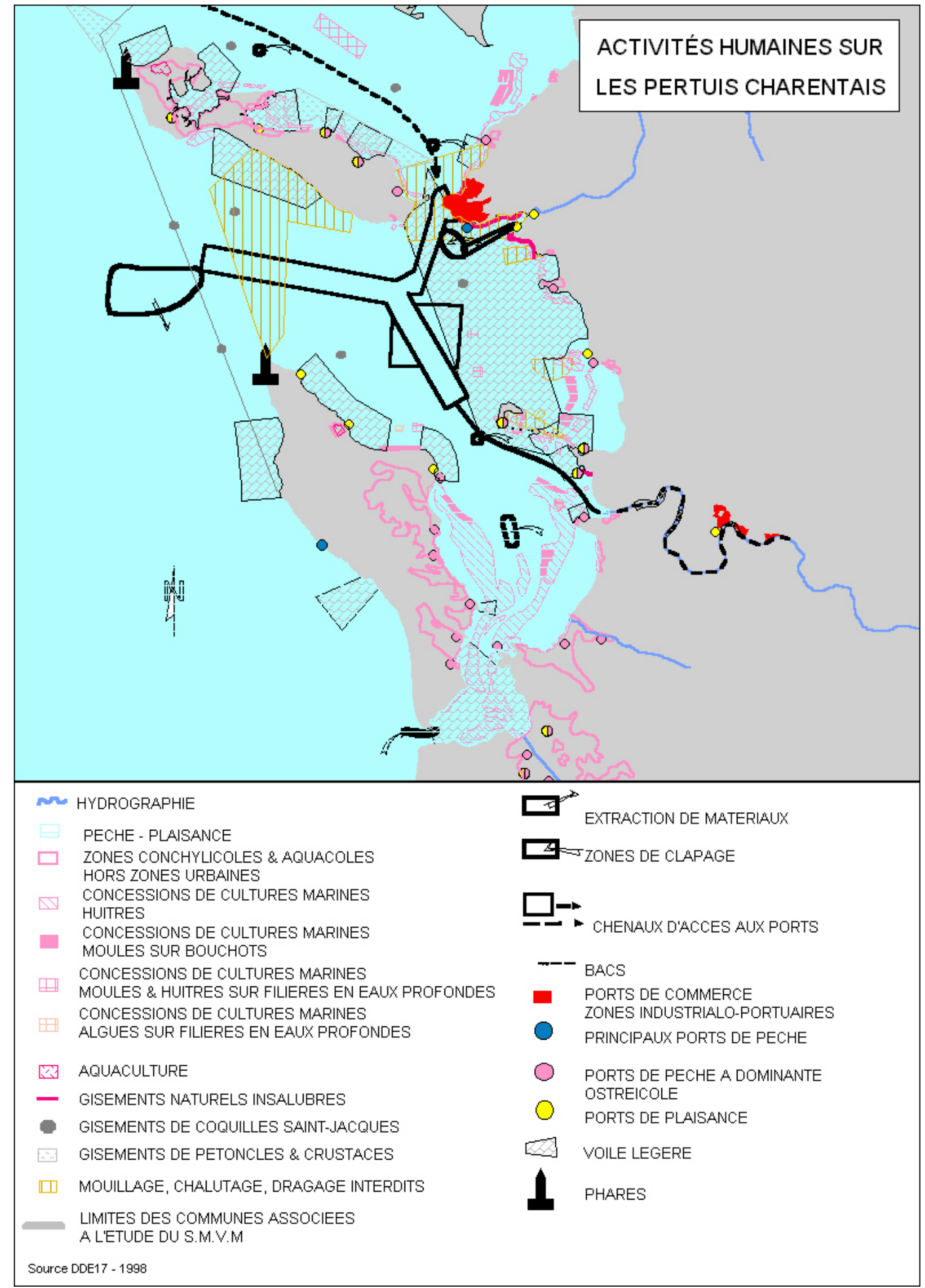

Figure 2: Activités humaines sur les pertuis charentais - DDE-17 1998

Schéma de Mise en Valeur de la Mer (S.M.V.M) source DDE 17 - 1998 


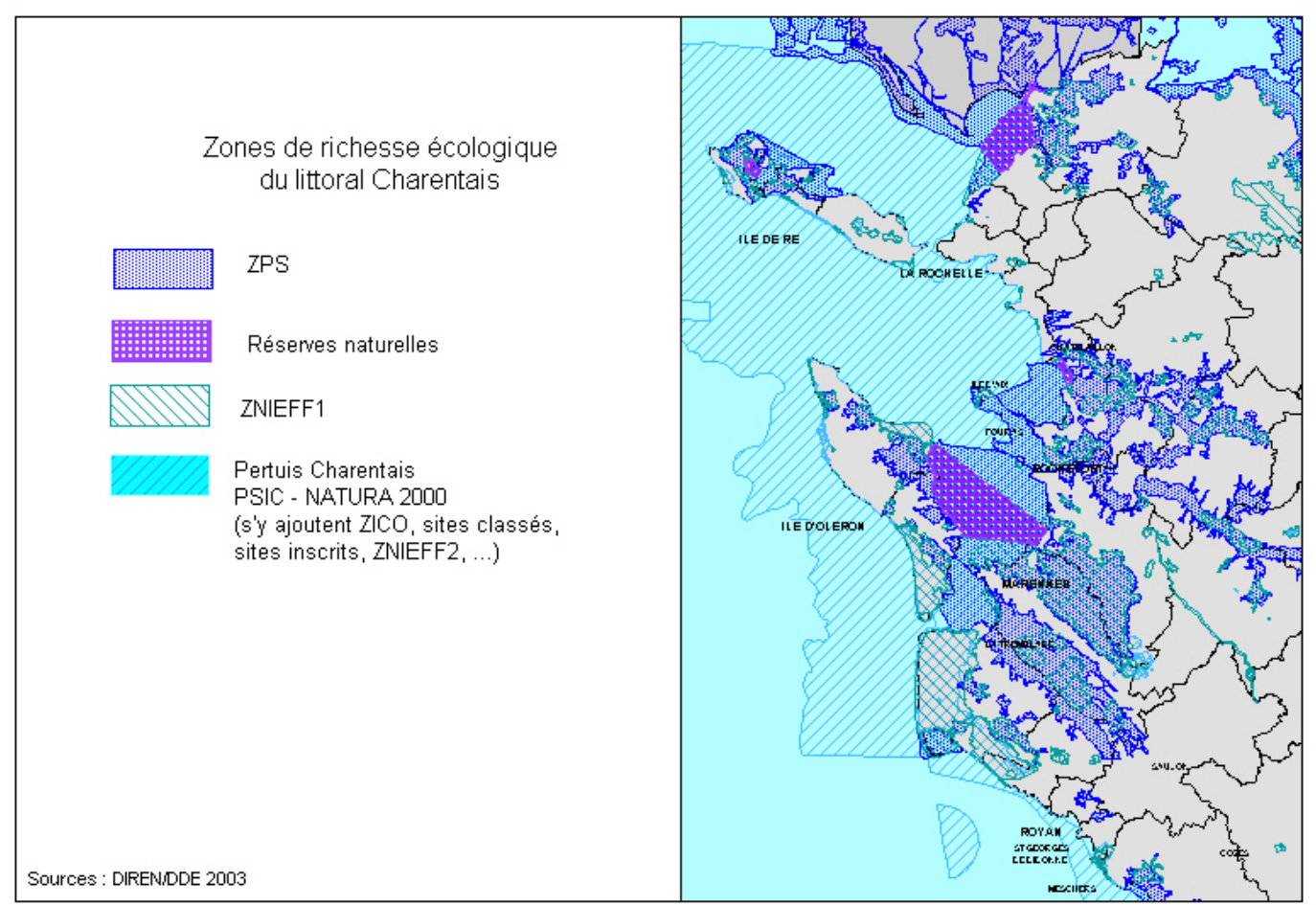

Figure 3: Zones de richesse écologique du littoral Charentais - DIREN/DDE-17

Protection des Espaces Naturels - Sources DIREN / DDE17 2003

\section{Démarche générale d'études sédimentologiques engagées sur le département de la Charente-Maritime en 1998}

Une étude générale financée par le Département de la Charente-Maritime et réalisée par la SOGREAH, a permis en 1995 de vérifier la cohérence générale du dispositif de défenses de côtes gérées par les collectivités publiques, et de disposer de préconisations pour chaque secteur du littoral.

Cette étude a notamment préconisé le principe de la défense des secteurs littoraux sableux (ou même à galets) soumis à érosion par des rétablissements du transit sédimentaire et des rechargements de plage en certains points stratégiques du littoral. Il s'agit de permettre à plusieurs côtes aujourd'hui sous-alimentées de retrouver un profil d'équilibre.

\subsection{Intérêt des rechargements de plage}

Ce type d'action apparaît particulièrement intéressant à développer pour plusieurs raisons : - En certains points du littoral charentais, on observe des accumulations de sables ( au niveau des ports, des chenaux de navigation, des zones d'approche des appontements pour vedettes en mer, des secteurs ostréicoles ) qui peuvent perturber l'activité économique associée ; ces sables sont alors dragués.

- Il serait très intéressant, lorsque cela est possible, de les mettre en dépôt sur des sites déficitaires en sable plutôt que dans des fosses marines de dépôt de produits de dragage courants.

- Les sites déficitaires sont notamment des côtes sableuses qui subissent une érosion ou qui sont à l'origine pauvres en sable. Un rechargement en sable constituera par lui-même une défense contre la mer et apportera une qualité améliorée des plages pour le tourisme. 
- Les premières opérations de rechargement ont été réalisées en 1990 sur Châtelaillon : 300 $000 \mathrm{~m} 3$ de sable. Depuis, ce sont plus de $500000 \mathrm{~m} 3$ de sable qui ont été mis en œuvre sur les plages de Marennes, Royan, Châtelaillon, Fouras. Sont également à citer les projets suivants, étudiés ou en cours d'études : rechargements plage nord de Châtelaillon et plage de Port-desBarques par du sable de dragage de la sortie du chenal de La Perrotine à Boyardville, rechargement côte sud de l'île de Ré par $300000 \mathrm{~m} 3$ de sable à draguer dans le chenal de navigation du Fier d'Ars, chargement côte nord d'Oléron par sable de vidage des pièges à sable du port de St Denis d'Oléron.

\subsection{Gestion dynamique des sédiments sur le littoral du département}

La démarche en cours a été engagée en 1998 par le Conseil Général de la CharenteMaritime sous l'impulsion du Syndicat des Pertuis, maître d'ouvrage pour des opérations de ré ensablement de plage.

Quatre grandes phases sont prévues :

\section{Phase I: (réalisée)}

La première phase, qui a fait l'objet d'un rapport remis par le CETMEF en avril 2000, a consisté en une exploitation bibliographique des principales études disponibles dans la littérature ( documents rassemblés auprès de la DDE 17, du CETMEF, du BRGM, et du SHOM )

Elle décrit à l'échelle départementale l'ensemble des transferts sédimentaires sableux sur les fonds et à la côte, identifiant en outre les principales zones d'engraissement et d'érosion associées. À partir de la description des différents transports sédimentaires, elle délimite également le littoral en 16 zones homogènes vis-à-vis du transit littoral. $\rightarrow \underline{\mathrm{Cf} \text { figure } 1 . \text { Elles }}$ obéissent aux critères suivants : homogénéité des zones par rapport au transit, pas ou peu d'interaction entre zones adjacentes. Il est à noter que la complexité des phénomènes naturels n'a pas permis de toujours respecter strictement ces deux conditions.

\section{Phase II: (réalisée pour partie)}

Sur la base de la délimitation réalisée en phase I, chaque zone fait ou va faire l'objet d'une étude particulière qui approfondit et précise au niveau local les résultats de la première phase. Les réflexions se concentrent notamment sur un certain nombre de points identifiés et rappelés en fin de phase I. Les études particulières doivent aboutir aux premiers éléments d'un plan de gestion des ressources sédimentaires ainsi qu'à des propositions d'aménagements axées sur l'utilisation de rechargements de plage et le rétablissement des transits sédimentaires.

Fin 2003, les études réalisées correspondent d'une part aux côtes ouest et nord de l'île d'Oléron (Bureau d'études Créocéan, avec connaissances complémentaires sur les fonds apportées par le CLDG de l'université de La Rochelle), et d'autre part aux côtes de l'île de Ré (bureaux d'études IN VIVO/SOGREAH, coordonnées avec les études menées par le CLDG).

En 2004, il est prévu le lancement d'une étude complexe sur le bassin de MarennesOléron et Pertuis de Maumusson (inter face sable/vase, intérêts socio-économiques, migration $\mathrm{du}$ pertuis, configuration des substratums, évaluation des stocks et mouvements sédimentaires, contraintes liées à la réserve naturelle...)

\section{Phase III:}

Les résultats des différentes études menées en phase II seront ensuite rassemblés et synthétisés sous forme d'un schéma global de gestion des sédiments à l'échelle du département.

Phase IV (déjà pour partie en cours):

La démarche globale s'achèvera par la finalisation et la mise en place d'un suivi morphologique global du littoral charentais à long terme destiné d'une part à mesurer 
l'efficacité des différents aménagements entrepris, et d'autre part à affiner les connaissances des processus hydrodynamiques et sédimentaires pour optimiser la conception des futurs aménagements et limiter toujours plus leur impact sur le milieu système (campagnes annuelles de levés et prélèvements pour l'évaluation des évolutions granulomètriques et topographiques des plages, corrélation entre l'environnement des plages et leur évolution temporelle).

Au croisement du contexte naturel et de la mise en valeur humaine du littoral, cette démarche de gestion intégrée se veut une démarche de développement durable, devant s’intégrer à la démarche plus générale de Gestion Intégrée des Zones Côtières.

\section{Contenus des premières études par zones, premiers résultats}

Les premières zones étudiées sont les côtes ouest et nord de l'île d'Oléron et les côtes de l'île de Ré, ainsi que les fonds marins qui leur correspondent. Elles comprennent :

- L'étude des transits côtiers avec l'utilisation de dispositifs de mesures tels que courantomètres, traçages fluorescents, houlographe directionnel, observations par satellite...

- L'étude des fonds, et en particulier des transports de sédiments sur ces fonds. Ont été utilisés des outils scientifiques comme le sonar latéral $\rightarrow \underline{\text { Cf figure } 4}$ sur le cas des côtes nord de l'île d'Oléron

- Un croisement de la nature de ces fonds, et des figures sédimentaires données par le sonar avec les résultats de campagnes de sismique profonde réalisées par le CLDG de l'Université de La Rochelle; ceci a permis de connaître en particulier le substratum et de consolider la compréhension du fonctionnement historique des transports sur les fonds.

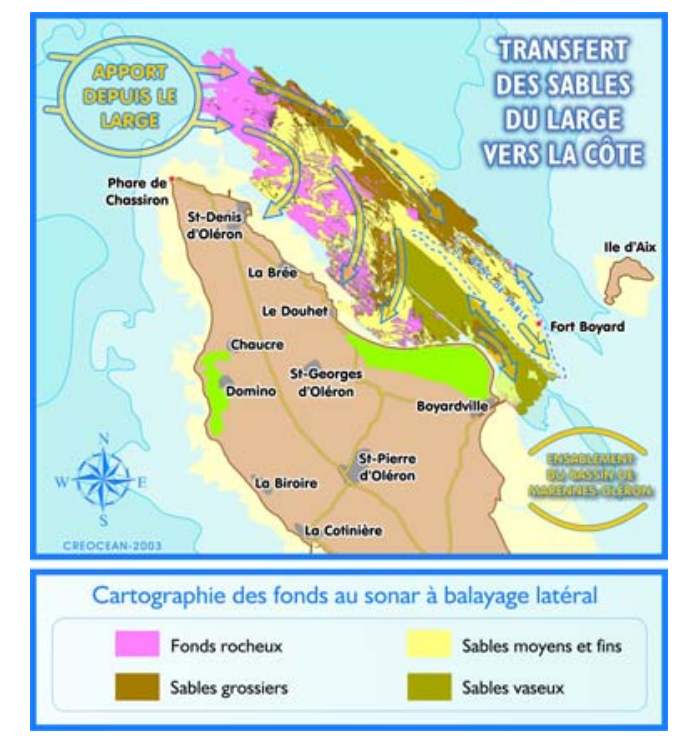

Figure 4: Transfert des sables du large vers la côte - CREOCEAN 2001

- L'évaluation des transports éoliens suivant un code de calcul développé par CREOCEAN sur la base de la formule de Bagnold par une simulation des transport en fonction des données de vents. Ceci permet de déterminer l'importance du débit solide associé s'exerçant sur les sables des plages (voir figure 5) pour l'île d'Oléron

- Des modèles mathématiques ont par ailleurs été utilisés 


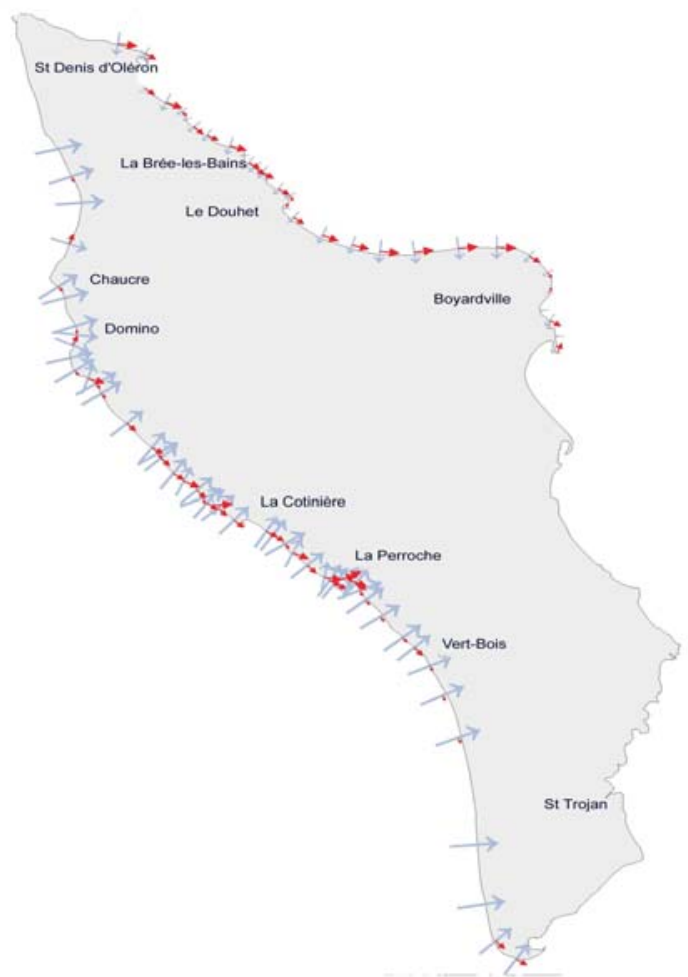

Figure 5: transport éolien (composante orthogonale à la côte en bleu, composante parallèle à la côte en rouge) - CREOCEAN 2001

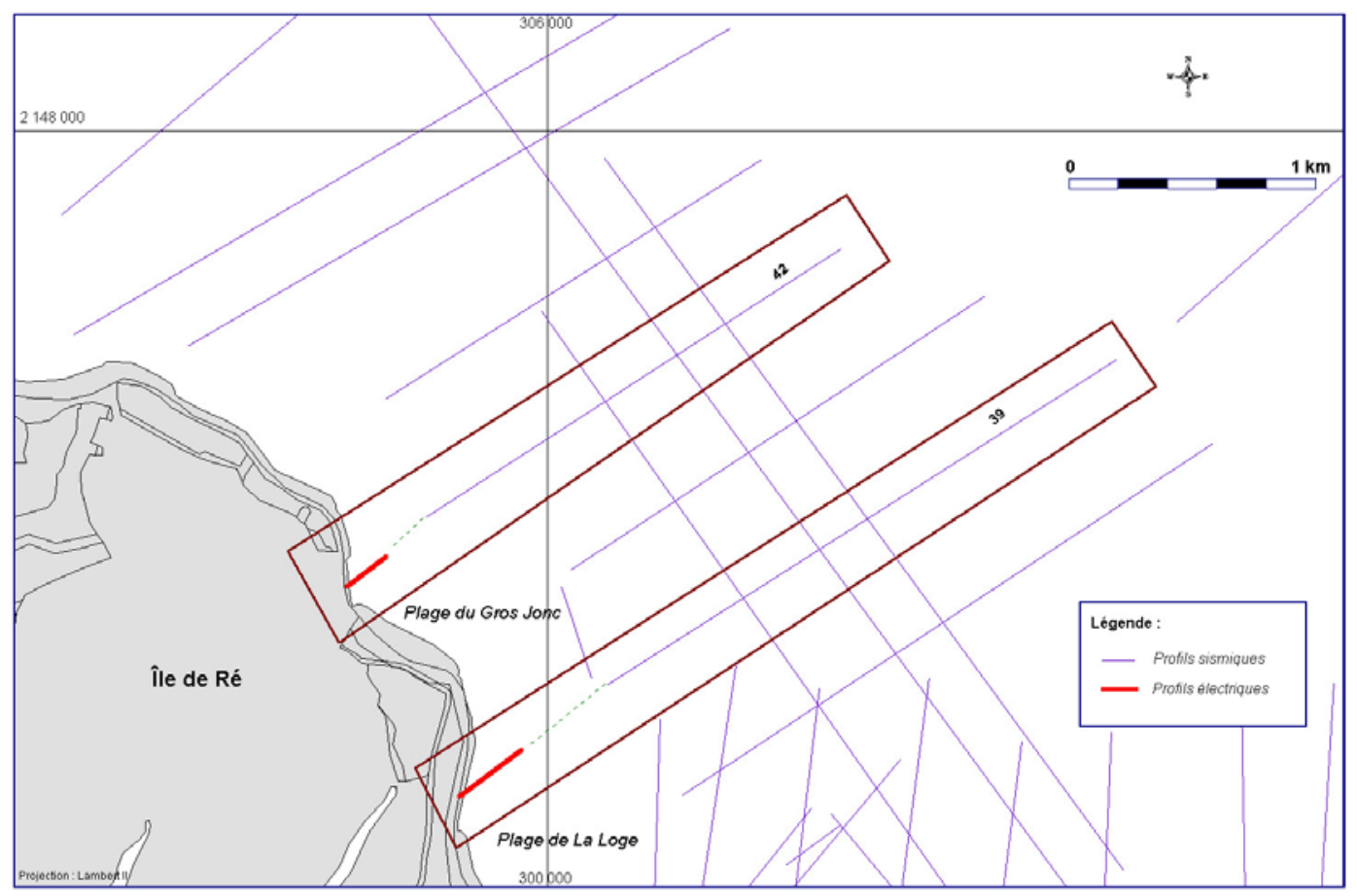

Figure 6: Mesure des épaisseurs de sable sur les estrans IN- VIVO/SOGREAH 2003 
La cohérence des résultats obtenus par les diverses méthodes employées ont fait l'objet de vérifications grâce à la coordination des approches: par exemple, les mesures des épaisseurs du sable sur les estrans par essais diélectriques ont été coordonnées avec les mesures par sismique sur les fonds marins (voir figure 6 ).

D'autres outils ou méthodes seront coordonnés pour la zone de Marennes-Oléron dont l'étude démarre en 2004 : on peut citer les investigations prévues sur les mers de vent, les courants de marée, la configuration des substratums, les effets des activités conchylicoles avec en particulier les bio-dépôts, les envasements avec des mesures de rhéologie, les transports éoliens.

\subsection{Quelques premiers résultats à ce jour}

Les connaissances se sont affinées sur les deux premières zones étudiées (Oléron ouest et nord, Ré) et ces connaissances ont particulièrement permis, dans le cadre de cette démarche de gestion opérationnelle, de définir des actions à lancer sur ces secteurs. A titre d'exemple:

- Sur la côte nord de l'île de Ré, au voisinage du banc du Bûcheron, les solutions possibles pour des transferts de sable sont indiquées (voir figure 7)

- Sur les côtes exposées au vent et à la houle du large de l'île d'Oléron, côtes sableuses pour la majeure partie, des dispositifs de pieux bois, ganivelles, et branchages, sont proposés, au regard des transits littoraux hydrauliques, des transports éoliens importants, et des dégradations dues à la fréquentation humaine(voir figure 8)

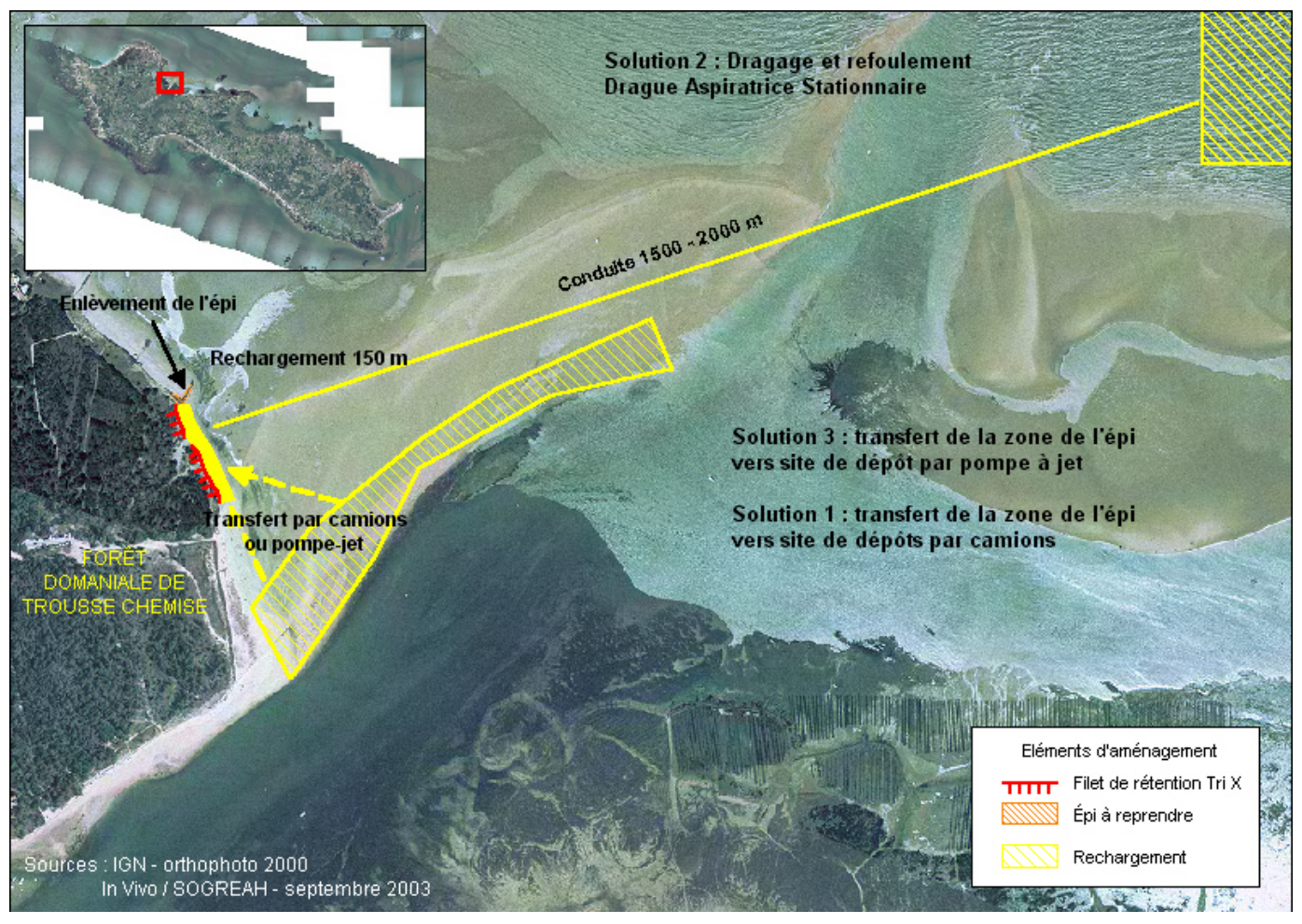

Figure 7: Transfert de sable - INVIVO/SOGREAH 2003 
Dispositif de piégeage de haute plage

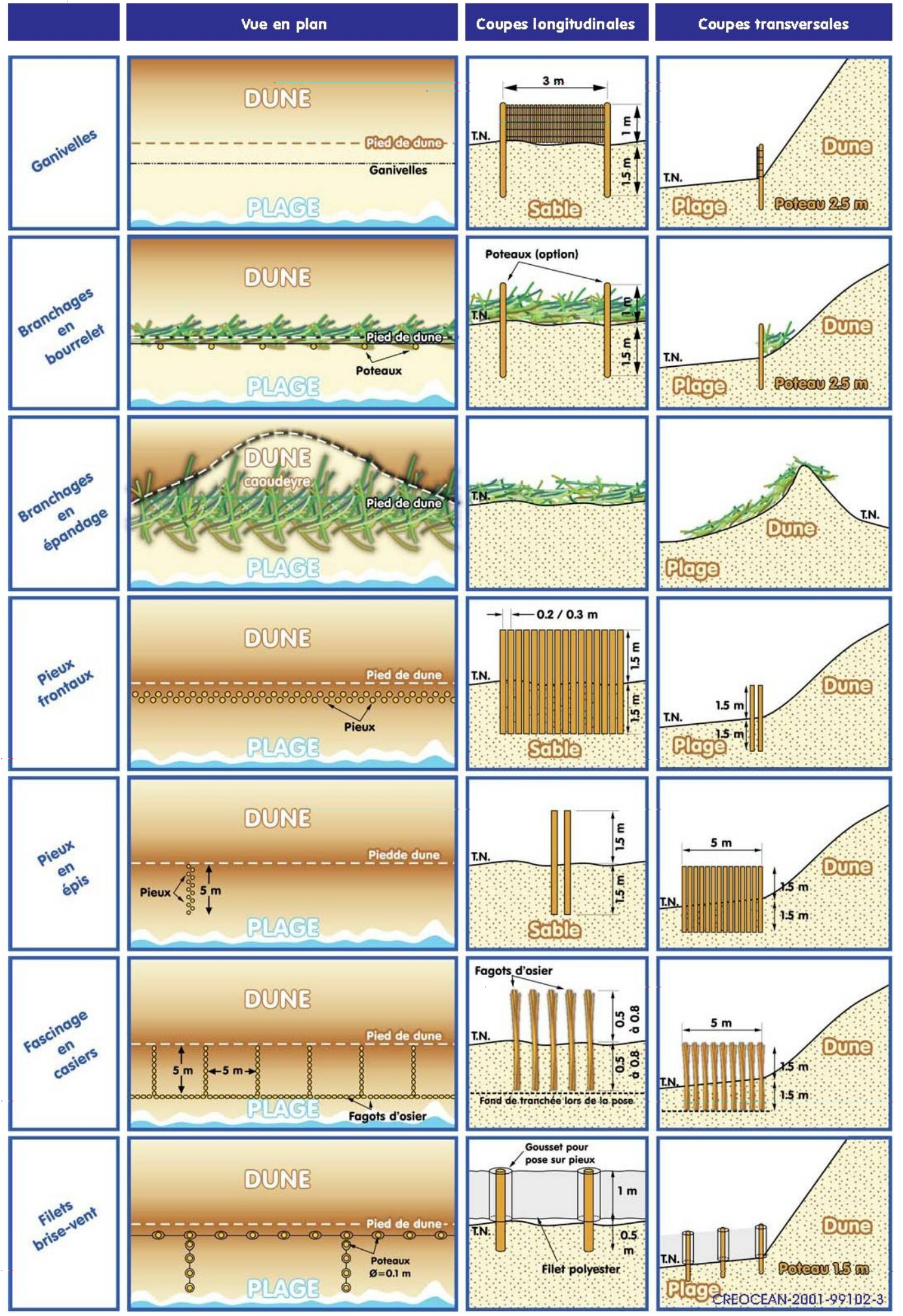

Figure 8: Dispositif de piégeage de haute plage - CREOCEAN 2001 
Les résultats attendus des études sur le bassin de Marennes-Oléron et le Pertuis de Maumusson devront permettre de définir et coordonner des actions de gestion intégrant la complexité de cette partie du littoral charentais, avec en particulier les systèmes de bancs et chenaux, les interactions entre sédiments vaseux et sableux, la forte évolution du delta de Maumusson, la très grande richesse écologique, la conchyliculture, et la navigation.

Enfin un plan de suivi des côtes a été défini pour chacun des secteurs dès le début, avec en particulier des profils de plages ; ce suivi sera prolongé et adapté en fonction des résultats des études par zones.

\section{Originalité et intérêts de la démarche}

La démarche suivie par le Conseil Général de la Charente-Maritime avec l'appui de la Direction Départementale de l'Équipement allie un développement des connaissances et la structuration d'une gestion intégrée et concertée sur l'espace littoral

- Les connaissances scientifiques et techniques de différentes disciplines sont recoupées et mises en valeur. Les transports de sédiments sont évalués sur les fonds marins comme sur les estrans, et en éolien.

- Le projet lui-même rassemble les conditions de la bonne utilisation des connaissances acquises ou à acquérir : cette gestion des sédiments associe nécessairement de nombreux partenaires, en particulier les différents niveaux de collectivités publiques. Les systèmes de défense douce sont recherchés en priorité ; la cohérence avec les Plans de Prévention des Risques Submersion/Erosion, et le respect de la protection des espaces, sont vérifiés.

\section{Références}

1.ANDRE X., Golfe de Gascogne, 1986, Elaboration et analyse de cartes bathymétriques détaillées du proche plateau Vendéo-Charentais - Reconstitution des paléorivages de la transgression Holocène, Université Bordeaux I, Thèse

2.BARUSSEAU J.P., Golfe de Gascogne, 1973, Evolution du plateau continental rochelais au cours du Pléistocène terminal et de l'Holocène, Université de Bordeaux I, Thèse

3.BCEOM, Gironde, 1988, Port de Plaisance de Saint Georges de Didonne, Etude d'impact.

4.BCEOM, Ile d'Oléron, 1987, Construction d'un port de plaisance - Commune de Saint Denis d'Oléron, Etude d'impact.

5.BIZIEN R., Pertuis de Maumusson, 1998, Evolution morphodynamique d'une passe tidale : le pertuis de Maumusson, 1 rapport, Bordeaux 1 / SHOM.

6.BRGM / IFREMER, Gironde, 1997, Elaboration d'un outil de gestion prévisionnelle de la côte aquitaine : phase 1, 1 rapport + plans.

7.BRGM, Ile d'Oléron, Carte au $8000^{\mathrm{ème}}$.

8.CHAUMILLON E., TESSON M., WEBER N., MAURIN J .C., Charente Maritime, 1999, Résultats préliminaires de la campagne de sismique haute résolution SIFADO soumis aux Cras,

9.CREOCEAN / CABINET GODARD - LAUNAY - MOREAU, Pertuis d'Antioche, 1995, Cartographie des colonies de crépidules entre Fouras, île d'Aix et La Rochelle, 1 rapport + 1 carte.

10.CREOCEAN / IFREMER, Marennes Oléron, 1995, Cartographie des colonies de crépidules dans le bassin de Marennes Oléron, 1 rapport +4 cartes.

11.CREOCEAN, Continent, 1997, Réensablement d'entretien de la Grande Plage Chatelaillon, Etude d'impact

12.CREOCEAN, Ile de Ré, 1997, Cartographie des colonies de crépidules - Secteurs NE et $\mathrm{SW}$ de l'île de Ré, 1 rapport +2 cartes. 
13.CREOCEAN, Ile de Ré, 1997, Cartographie des colonies de crépidules des côtes S et N de l'île de Ré, 1 carte.

14.CREOCEAN, Ile de Ré, 1997, Rejets de produits de dragage des ports de Saint Martin de Ré, Ars en Ré, La flotte en Ré sur la fosse orientale de Chevarache, Etude d'incidence.

15.CREOCEAN, Pertuis breton, 1996, Rejet de produits de dragage sur différents sites dans le pertuis breton, Etude de faisabilité.

16.CREOCEAN, Pertuis Breton, 1998, Cartographie des colonies de crépidules - zone méridionale du pertuis breton, 1 rapport +2 cartes.

17.DUBOUL - RAVAZET, BARUSSEAU C., LONG B., Ile de Ré, 1972, Rapport préliminaire concernant le banc du Bûcheron, Rapport, Centre de recherches de sédimentologie marine de Perpignan.

18.GEOMARINE, Ile d'Oléron, 1991, Evolution de la pointe de la Gautrelle, 1 rapport.

19.LAFOND, Gironde, 1992, Les problèmes sédimentologiques dans la zone de la Coubre, 1 rapport, E.P.H.E.

20.LCHF, BELLESORT B., MIGNIOT C., Charente Mar., 1987, Catalogue sédimentologique des côtes françaises sous la maîtrise d'ouvrage du STCPMVN (CETMEF)

21.LCHF, Charente Maritime, 1979, Littoral de la Charente Maritime - Etude prospective de défense des côtes, 1 rapport.

22.LCHF, Pertuis, 1969, Pertuis breton et d'Antioche - Etude de synthèse, rapport.

23.LE ROUX G., Pertuis charentais, 1996, Information géographique et défense du trait de côte sur les Pertuis Charentais - Etude de faisabilité d'un SIG - Application à la zone test de l'île de Ré, 1 rapport + 1 annexe cartographique, IFREMER - IFEN - ESEM.

24.LECLER, Charente Mar., 1970, Le recul des côtes de Charente Maritime, 1 rapport

25.LONG B., Ile de Ré, 1975, Le Littoral NW de l'île de Ré : Les processus dynamiques de la sédimentation et l'évolution côtière récente, Thèse

26.LONG B., Ile de Ré, 1972, Rapport préliminaire sur le Banc du Bûcheron, rapport d'études.

27.MALLET C., Gironde, 1998, Etude de la dynamique des sédiments non cohésifs de l'embouchure de la Gironde, Université de Bordeaux I, Thèse

28.MARENDET F., RAVARD P., MIOSSEC A., LAFOND R., SORNIN J.M., BELLESSORT B., Ile de Ré, 1992, Aménagement de l'espace côtier de l'île de Ré, rapport comité scientifique.

29. OBSERVATOIRE DU LITTORAL, Gironde, 1998, Communauté de Communes du Pays Royannais, 1 rapport

30.PIGNON C., Ile de Ré, juillet 1996, Réhabilitation du fiers d'Ars et Protection des plages de l'île de Ré, Etude de financement.

31.COMPAGNIE ARMORICAINE DE NAVIGATION/RAMBAUD CARRIERES, Ile d'Oléron, 1996, Gisement de sables et graviers siliceux marins Chassiron «C »- Demande d'autorisation d'exploitation (concession), Etude d'impact, Compagnie armoricaine de navigation Rambaud Carrières.

32.CREOCEAN, Ile d'Oléron, 1998, Gisement de sables et graviers siliceux marins Chassiron «B »- Demande d'autorisation d'exploitation (concession), Etude d'impact, Granulats Ouest DTM.

33.RIVAGES, Fouras, mars 1999, Etude technico-économique des conditions de rechargement des plages $\mathrm{W}$ et $\mathrm{N}$ de la commune de Fouras, rapport provisoire

34.CEDRE, Charente Mar., septembre 1986, Atlas pour la protection et la lutte contre les pollutions accidentelles venant de la mer, des Sables d'Olonne à l'entrée de la Gironde Pertuis charentais, rapport

35.SERVICE MARITIME DE CHARENTE MARITIME, Baie de Marennes Oléron, 1997, Etude hydrographique, Rapport Cellule Hydrographique. 
36.SHOM, Charente Mar., 1998, Réalisation de la carte sédimentologique 6333G «De l'île de Ré à l'île d'Oléron », rapport.

37.SHOM, Charente Mar., 1999, Réalisation de la carte sédimentologique 7405 G, en préparation.

38.SOGREAH, Charente Mar., 1987, Etude prospective du littoral de la Charente Maritime : Synthèse, tome 6.

39.SOGREAH, Charente Mar., 1995, Etude de défense des côtes, 1 rapport + annexe cartographique

40.SOGREAH, Continent, 1987, Etude prospective du littoral de la Charente Maritime : Baie d'Yves, tome 3.

41.SOGREAH, Gironde, 1987, Etude prospective du littoral de la Charente Maritime : Aménagement du littoral La Coubre Saint-Palais, tome 4.

42.SOGREAH, Ile d'Oléron, 1987, Etude prospective du littoral de la Charente Maritime : Aménagement du littoral de l'île d'Oléron, tome 5.

43.SOGREAH, Ile de Ré, 1987, Etude prospective du littoral de la Charente Maritime: Fiers d'Ars, tome 1.

44.SOGREAH, Ile de Ré, 1987, Etude prospective du littoral de la Charente Maritime : Ile de Ré, tome 2.

45.SOGREAH, Ile de Ré, 1991, Création d'un bassin de chasse et extension du port de plaisance d'Ars en Ré, Etude d'impact.

46.TESSON M., Marennes Oléron, 1973, Aspects dynamiques de la sédimentation dans la baie de Marennes Oléron, Université de Bordeaux I, Thèse.

47.VERHELlE A., Charente Mar., 1994, Etude de faisabilité de l'utilisation d'un SIG comme outil d'aide à la décision dans l'évaluation du risque d'érosion et de submersion de la bande littoral, 1 rapport + annexe cartographique, ESEM/BRGM.

48.VERHELLE A., Charente Mar., 1994, SIG et érosion côtière, Rapport, ESEM.

49.VERHILLE S., Charente Mar., 1998, Gestion dynamique des sédiments sur le domaine public maritime le long du littoral charentais, Rapport de stage, DEUST (Côte d'Opale).

\section{références complémentaires: ouvrages à exploiter lors des études zone par zone (d'après} recherches C. MIGNIOT)

50.ALLEN G., 1972, Estuaire de la Gironde, Thèse, Bordeaux 1

51.BARUSSEAU J.P., 1965, Contribution à l'étude des sédiments de la Charente Maritime, DES Montpellier,

52.BARUSSEAU J.P., 1967, Etude granulométrique des sédiments des pertuis charentais, Cahiers Océanographiques T 19,

53.BAXERRES P., 1978, Etude morphologique et sédimentologique de la côte atlantique de Saintonge de la pointe sud de l'île d'Oléron à la pointe de la Coubre, thèse, Bordeaux 1,

54.BCEOM, 1973, Le littoral français- dommages côtiers- Division Port et Nature

55.BOUQUET de la GRYE, 1877, Le régime hydrographique des pertuis de la Charente Maritime, Journ. Hist. Nat. , T 1

56.De ROUVILLE A., 1942, Le régime des côtes, Imprimerie Nationale,

57.FERAL A., 1970, Interprétation sédimentologique et paléographique des formations alluviales flandriennes de l'estuaire de la Gironde et de ses dépendances marines, Thèse, Bordeaux,

58.GALICHON P., 1984, Hydrodynamique sédimentaire des flèches littorales - La cas de la pointe d'Arcay, Thèse Paris sud

59.GERMANEAU J.,1977, Etudes des sables littoraux de l'île de Ré, Travaux du Centre de Recherche et d'Etudes Océanographiques, vol 17, fasc. 1 et 2 
60.LALAUT Y., 1992, Fonctionnement et efficacité des ouvrages statiques de protection des littoraux sableux, Université Paris Sud, Thèse,

61.LCHF, 1956, Pointe des Sablanceaux, Mission en Nature et modèle réduit sédimentologique, DDE/LCHF,

62.LCHF, 1958, La Cotinière - Mission d'étude en Nature, DDE/LCHF

63.LCHF, 1959, Royan, Mission d'étude en nature, DDE/LCHF,

64.LCHF, 1960, Le Douhet, Etude en Nature, Commune de Saint Georges/LCHF

65.LCHF, 1962, Le Douhet, Etude sur modèle réduit, Commune de Saint Georges/LCHF

66.LCHF, 1963, Chatelaillon, Mission d'études en Nature, DDE/LCHF

67.LCHF, 1966, Les Minimes, Port de Plaisance, Mission d'étude en Nature, DDE/LCHF

68.LCHF, 1973, La Coubre, Etude en Nature- SIVOM/LCHF

69.LCHF, 1977, La Cotinière, Extension du Port, Commune de Saint Pierre/LCHF

70.LCHF, 1977, La Rochelle - La Pallice, Extension du port - Conditions naturelles, DDE/LCHF

71.LCHF, 1977, Marennes, Aménagement de la plage - Avis Technique DDE/LCHF/Ville de Marennes

72.LCHF, 1978, La Coubre - Saint Palais, Etude sur modèle physique

73.LCHF, 1978, Malconche, Défense des côtes de l'anse de Malconche,

74.LCHF, 1979, Port Neuf, Aménagement de l'anse de Port Neuf, DDE/LCHF,

75.LCHF, 1979, Royan, Base Nautique et remodelage du tracé de la plage, SEMIPAR/LCHF,

76.LCHF, 1982, Saint Denis d'Oléron, Aménagement d'un port de plaisance, DDE/LCHF

77.LCHF, 1977, Les Portes en Ré, Erosion de la côte, Avis technique, DDE/LCHF

78.LORIN J., 1968, Contribution à l'étude des transits sédimentaires de la pointe orientale du pertuis breton et de la baie de l'Aiguillon, Bull. Inst. Géol. Bassin Aquitain $\mathrm{n}^{\circ}$ 9,

79.MASSON P., 1979, Contribution à l'étude sédimentologique des côtes charentaises (estuaire de la Gironde, presqu'île d'Arvert, île d'Oléron), thèse, Paris sud

80.SOGREAH, 1980, Port de Saint Denis d'Oléron - Faisabilité du futur port - DDE Charente Maritime

81.SOGREAH, 1986, Port de Saint Denis d'Oléron, Optimisation du programme sur le plan sédimentologique

82.SOGREAH, 1987, Actualisation de l'état du littoral charentais - Evolution - Travaux à envisager

83.SOGREAH, 1988, Ville de Royan, Port de Royan - Etude de préfaisabilité de l'extension du Port de Royan - Agitation et sédimentologie

84.SOGREAH, 1988, Port de la Perruche - Préfaisabilité, commune de Dolus, Charente Maritime

85.SOGREAH, 1989, Ouvrage de défense du littoral de la Palmyre - DDE Charente Maritime - Subdivision de Royan

86.SOGREAH, 1989, Port de plaisance des Minimes - DDE Charente maritime 